\title{
CORM-2 can Attenuate Bleeding-mediated Inflammation by Increasing Phagocytic Capacity of Cerebral Microglial Cells in Neonatal Rat in Vitro
}

Zhiying Chen ( $\nabla$ chenzhiying@ccmu.edu.cn )

Capital Medical University https://orcid.org/0000-0001-6020-4484

Huiyan Zhang

Jiujiang University

Jun Zhou

Shanghai 6th Peoples Hospital Affiliated to Shanghai Jiaotong University School of Medicine

Xiaoqin Wu

Capital Medical University

Moxin Wu

Jiujiang University

Ling He

Jiujiang University

Xiaoping Yin

Jiujiang University

Ran Meng

Xuanwu Hospital

\section{Research Article}

Keywords: CORM-2, Microglia, inflammation, NF-kB

Posted Date: March 19th, 2021

DOI: https://doi.org/10.21203/rs.3.rs-313030/v1

License: (1) (1) This work is licensed under a Creative Commons Attribution 4.0 International License.

Read Full License 


\section{Abstract}

Objective: This study aimed to explore the mechanism of CORM-2 on attenuating bleeding-related inflammation.

Methods: Microglia were isolated from the neonatal rats (1-2days old) and identified by the CD11b/c antibody, and some microglia were co-cultured with RBCs marked with PKH26 fluorescent dye, and then treated with CORM-2. That is, the microglia cells were divided into the microglia, microglia+ ${ }^{\mathrm{PKH} 26+} \mathrm{RBCs}$ and microglia $+{ }^{\mathrm{PKH} 26+} \mathrm{RBCs}+\mathrm{CORM}-2$ cell-groups. Microglial phagocytosis to $\mathrm{RBCs}^{\mathrm{PKH} 26+}$ was observed under an inverted fluorescence microscope; moreover, the fluorescence intensity of microglia that phagocytized ${ }^{\mathrm{PKH} 26+} \mathrm{RBC}$ was detected through immunofluorescence. HO-1, NF-KB p65, and IL-1 $\beta$ expressions were detected using RT-qPCR, western blotting, and immunofluorescence, respectively. The levels of carbon monoxide hemoglobin $(\mathrm{HbCO})$ in the cell supernatant in each group were detected with ELISA.

Results. After 1- day of co-culturing, the number of residual ${ }^{\mathrm{PKH} 26+}{ }^{\mathrm{RBC}}$ in the Microglia+ $\mathrm{PKH}^{26+} \mathrm{RBCs}+\mathrm{CORM}-2$ group decreased remarkably than that in the Microglia+ ${ }^{\mathrm{PKH} 26+} \mathrm{RBC}$ groups $(18 \times$ $10^{6}$ vs. $\left.14 \times 10^{6}, \mathrm{p}=0.02\right)$, which revealed that microglia phagocytosis was stronger in CORM-2 treated group. More over, compared with microglia $+{ }^{\mathrm{PKH} 26+} \mathrm{RBC}$ group, the microglia $+{ }^{\mathrm{PKH} 26+} \mathrm{RBC}+\mathrm{CORM}-2$ group showed higher levels of HO-1 mRNA and protein expressions at the $3^{\text {rd }}$ day and the $5^{\text {th }}$ day after coculturing. Further more, CORM-2 significantly inhibited the expressions of mRNA and proteins of NF-KB p65 and IL- 1 after 3 days of co-culturing, meanwhile, CORM-2 did not increase the level of $\mathrm{HbCO}$ in the cell supernatant.

\section{Conclusions}

CORM-2 can inhibit inflammatory reactions in bleeding setting in vitro by promoting microglial phagocytosis to RBCs and decrease IL-1 $\beta$ and NF-KB; the mechanism may involve HO-1/CO system.

\section{Introduction}

Intracerebral hemorrhage $(\mathrm{ICH})$ is one of the most fatal stroke subtypes and lacks an effective medical treatment. The predominant hematoma component is red blood cells (RBCs), which are cleaved into thrombin and hemoglobin[1, 2]. Microglia clear and degrade hemoglobin into heme. In the presence of oxygen and nicotinamide adenine dinucleotide phosphate, human heme oxygenase $(\mathrm{HO})$ catalyzes heme oxidation resulted in a-meso-hydroxyheme and verdoheme, and finally formed biliverdin[2]. Hematomas release numerous inflammatory factors and chemokines, which have been considered as the main factors resulting in secondary brain injury $[1,3,4]$. Effectively inhibiting inflammatory response in perihematoma area may reduce $\mathrm{ICH}$-mediated secondary brain injury. 
Microglia are the first kind of non-neuronal cells involved in the innate immune response to $\mathrm{ICH}$. In acute brain injury, microglia can be activated and polarized into two phenotypes: M1-type (pro-inflammatory) and M2-type (anti-inflammatory) microglia [5, 6]. This implies microglia involvement in ICH pathological processes of both injury and repair. However, the mechanisms remain unclear, which may involve microglial production of pro-/anti-inflammatory cytokines and chemokines. Furthermore, targeted M2-likemicroglia activation may promote its phagocytosis of RBCs and tissue debris, which may benefit to hematoma clearance.

HO-1 mainly expressed in microglia/macrophages and endothelial cells post-ICH[7]. We found previously that $\mathrm{HO}$-1 up-expression could improve neuro-motor function, attenuate peri-hematoma inflammatory injury, and decrease NF-KB expression in a rat brain hemorrhage model [8-10]. In the lipopolysaccharide (LPS)-induced inflammatory injury model, sulforaphane help microglia exerted anti-inflammation effect by increasing the expression of HO-1 in microglia [11]. Another study also revealed that HO-1/CO system was closely related to the migration, phagocytosis, and anti-inflammation ability of microglia, including HO-1 promoted $\mathrm{CO}$ expression, which in turn increased HO-1 expression, to active this positive feedback $\mathrm{HO}-1 / \mathrm{CO}$ system could promote migration and the immune phagocytic function of microglia, and decrease the inflammatory factor expression[12], which may exert protective effects in central nervous system.

$\mathrm{CO}$, an endogenous antioxidant gas molecule produced by HO-1-mediated heme decomposition, exerts strong antioxidant effects. CORM-2 is a carbon-based compound that can slowly release CO, and lowlevel of $\mathrm{CO}$ is good for anti-inflammation and anti-microbe [13-15]. CORM-2 upregulates HO-1 to exert cytoprotective effect, and inhibits the activity of NF-KB [16]. However, the underlying molecular neuroprotective mechanism post-ICH of the $\mathrm{HO}-1 / \mathrm{CO}$ system remains unclear.

Herein, we aimed to explore the effect of $\mathrm{HO}-1 / \mathrm{CO}$ system on promoting microglia phagocytosis, as well as the possible mechanism of the $\mathrm{HO}-1 / \mathrm{CO}$ system on attenuating bleeding induced inflammation.

\section{Materials And Methods}

\section{Experimental reagents}

Goat Anti-Rabbit IgG/488 (ZF-0511, Beijing ZSGB-BIO Co., Ltd., Beijing, China); Anti HO-1 antibody (ab13243, abcam, Cambridge, UK); Anti IL-1 $\beta$ antibody (ab9722, abcam, Cambridge, UK); Anti CD11b/c antibody (ab33827, abcam, Cambridge, UK)Ultrapure RNA extraction kit (CW0581M, Beijing ComWin Biotech Co., Ltd., Beijing, China); HiFiScript cDNA synthesis Kit (CW2569M, Beijing ComWin Biotech Co., Ltd., Beijing, China); UltraSYBR Mix (CW0957M, Beijing ComWin Biotech Co., Ltd., Beijing, China); Fluorescent quantitative PCR instrument (CFX Connect ${ }^{\mathrm{T}}$, Bio-Rad Shanghai Laboratories, Shanghai, China); RIPA Cell lysis buffer (C1053, Beijing applygen Co., Ltd., Beijing, China); BCA Protein Assay Kit (CW0014S, Beijing ComWin Biotech Co., Ltd., Beijing, China); polyvinylidene fluoride (PVDF) membrane (IPVH00010, Millipore, Billerica, MA, USA); Rabbit Monoclonal Anti-GAPDH(TA-08, Beijing ZSGB-BIO Co., 
Ltd., Beijing, China); Anti NF-kB p65 (8242S, CST); Horseradish Enzyme Labeled Goat Anti-Rat IgG $(\mathrm{H}+\mathrm{L})$ (ZB-2305, Beijing ZSGB-BIO Co., Ltd., Beijing, China); Microplate Reader (RT-6100, Rayto, Shenzhen, China); Protein vertical electrophoresis instrument (DYY-6C, Beijing 61 instrument factory, Beijing, China).

\section{Microglia isolation and identification}

Five Sprague-Dawley (SD) neonatal rats (1-2 days old) were disinfected using $75 \%$ alcohol, decapitated, and their brains removed in a sterile environment. The isolated cerebral cortex was placed in a centrifuge tube with $2 \mathrm{ml}$ of Hanks solution and digested in $0.25 \%$ trypsin for 10 min. Digestion was ended with DMEM/F12 medium containing 10\% fetal bovine serum (FBS); subsequently, primary mixed glial cells were obtained through filtration and centrifugation. Microglia were isolated from shaking the petri dishes for $12 \mathrm{~h}$, and were identified with CD11b/c immunofluorescence test.

\section{Red blood cells isolation and labeled}

$1.0 \mathrm{ml}$ of blood was collected from the left heart ventricle of $5 \mathrm{SD}$ neonatal rats $(0.2 \mathrm{ml}$ / each rat) and placed in an acid-citrate-dextrose anticoagulant solution, followed by centrifugation at $400 \mathrm{~g} \times \mathrm{g}$ for 5 min for RBC collection. Washing the RBCs $\left(2 \times 10^{7}\right)$ thrice with a serum-free culture medium and then centrifuging them at $400 \times \mathrm{g}$ for $5 \mathrm{~min}$ to form a loose cell mass, and removing the supernatant, finally, the RBCs were suspended in $1.0 \mathrm{ml}$ of diluent to ensure complete dispersion. The RBC suspension was promptly added into the diluted dye solution $\left(4 \times 10^{-6} \mathrm{~mol} / \mathrm{L} \mathrm{PKH-26)}\right.$, mixed them with a pipette evenly and rapidly, and incubated at $25^{\circ} \mathrm{C}$ for 2 to $5 \mathrm{~min}$, added the same amount of FBS incubated for 1 min to stop the staining reaction, then centrifuged at $400 \times \mathrm{g}$ for $10 \mathrm{~min}$, removed the supernatant, the labeled RBCs were washed thrice with serum-containing medium to obtain ${ }^{\mathrm{PKH} 26+} \mathrm{RBCs}$.

\section{Co-culture of RBCs and microglia}

Microglia and ${ }^{\mathrm{PKH} 26+} \mathrm{RBC}$ were mixed at a $1: 10$ ratio and co-cultured in $37^{\circ} \mathrm{C}$ with $5 \% \mathrm{CO}_{2}$ for 1 day, meanwhile, some of them were treated with CORM-2 $(50 \mathrm{Um})$, to get the followings cell-groups: Microglia, Microglia $+{ }^{\mathrm{PKH} 26+} \mathrm{RBC}$, and Microglia $+{ }^{\mathrm{PKH} 26+} \mathrm{RBCs}+\mathrm{CORM}-2$. At the 6 th $\mathrm{h}$ and the 24 th $\mathrm{h}$ and the $3 \mathrm{rd}$ day after co-cultivation, respectively, the phenomenon of microglial phagocytosis to the ${ }^{\mathrm{PKH} 26+} \mathrm{RBCs}$ would be observed under the inverted fluorescence microscope.

\section{Immunofluorescence}

After fusion to $80 \%-90 \%$, cells in the cell-groups mentioned above were harvested and washed thrice with phosphate-buffered saline (PBS). The cells were fixed with $4 \%$ paraformaldehyde for $15 \mathrm{~min}$ and washed thrice with PBS. Next, $0.5 \%$ Triton X-100 was added for $20 \mathrm{~min}$. After $30 \mathrm{~min}$ of blocking with $5 \%$ bovine serum albumin at $37^{\circ} \mathrm{C}$, the cells were incubated with fluorescein isothiocyanate-conjugated primary antibody at $4^{\circ} \mathrm{C}$ overnight. Cy3-conjugated secondary antibody diluted with blocking buffer was used for staining at $37^{\circ} \mathrm{C}$ for $30 \mathrm{~min}$. Next, the cells were incubated with DAPI in the dark for $5 \mathrm{~min}$. The samples were sealed with $50 \%$ glycerol and observed under a fluorescence microscope. The expression of $\mathrm{HO}-1$, 
NF-Kb p65, and IL-1ßin all cell-groups at the 1st, 3rd, and 5th day were compared with RT-PCR, Western blot, ELISA, respectively.

\subsection{RT-qPCR}

Total RNA was extracted using an Ultrapure RNA kit (Takara, Tokyo, Japan) according to the manufacturer's protocol and subjected to reverse transcription using a HiFiScript cDNA Synthesis Kit. Real-time PCR was performed using SYBR Green PCR Master Mix. The $\beta$-actin gene was used as an internal control to normalize RNA quantity and quality differences in all samples. Target gene mRNA quantification was performed using the $2^{-\triangle} \triangle$ Ct method. Table 1 shows the primer sequences.

Table 1

Features of primers and products as well as annealing temperatures in this study

\begin{tabular}{|c|c|c|c|c|}
\hline $\begin{array}{l}\text { Primer } \\
\text { Appellations }\end{array}$ & Sequences & $\begin{array}{l}\text { Primer } \\
\text { Length } \\
\text { (bp) }\end{array}$ & $\begin{array}{l}\text { Product } \\
\text { length(bp) }\end{array}$ & $\begin{array}{l}\text { Annealing } \\
\text { temperature }\left({ }^{\circ} \mathrm{C}\right)\end{array}$ \\
\hline$\beta$-actin $F$ & GCCATGTACGTAGCCATCCA & 20 & 375 & 59.5 \\
\hline$\beta$-actin $\mathrm{R}$ & GAACCGCTCATTGCCGATAG & 20 & & \\
\hline $\mathrm{HO}-1 \mathrm{~F}$ & AGGTCCTGAAGAAGATTGCG & 20 & 279 & 59.0 \\
\hline HO-1 R & GGCGAAGAAACTCTGTCTGTGA & 22 & & \\
\hline NF-kB p65 F & ССТTTTCTCAAGCCGATGT & 19 & 207 & 55.7 \\
\hline NF-kB p65 R & CGTAGGTCCTTTTGCGTTT & 19 & & \\
\hline IL-1 $1 \beta \mathrm{F}$ & CAGACCCCAAAAGATTAAGGATTG & 24 & 263 & 57.6 \\
\hline IL-1 $\beta$ R & CTAGCAGGTCGTCATCATCC & 20 & & \\
\hline
\end{tabular}

\subsection{Western blot}

Protein extraction was performed for the different cell groups. Briefly, the cells were harvested and lysed, followed by protein extraction. Proteins were quantified using the BCA kit and loaded in SDS-PAGE gel (12\%, 5-10 $\mu \mathrm{g}$ per lane); subsequently, they were electrotransferred to a PVDF membrane. The membrane was rinsed and placed in blocking buffer. Samples were incubated at $4^{\circ} \mathrm{C}$ overnight after adding the primary antibodies. Next, the membrane was rinsed and incubated with a secondary antibody at room temperature for $2 \mathrm{~h}$. Photoelectrons were captured using the ImageQuant LAS 4000 imaging station (GE) followed by quantification.

\subsection{ELISA}

The levels of $\mathrm{HbCO}$ expression were determined with ELISA kit according to the manufacturer's instructions. The kit employs a double-antibody sandwich method. Purified rat HbCO was used for 
antibody capturing and coated with a microporous plate to make a solid-phase antibody. The cell supernatant was sequentially added to the coated microporous, followed by the addition of the horseradish peroxidase-labeled detection antibody to form an antibody-antigen-enzyme antibody complex. The absorbance (optical density value) was measured at a 450-nm wavelength using a microplate reader; moreover, the $\mathrm{HbCO}$ level in the sample was calculated from the standard curve.

\section{Statistical analysis}

Statistical analyses were performed using SPSS 22.0(IBM SPSS 22.0, SPSS Inc). All data were presented as the mean \pm standard deviation (SD). Between-group differences were evaluated using a one-way analysis of variance. Statistical significance was set at $P<0.05$.

\section{Results}

\section{Microglia identification}

To identify microglia among the isolated primary cells, CD11b/c expression was detected using immunofluorescence. As shown in Fig. 1, cells with positive CD11b/c (Green fluorescence) were identified as microglia.

\section{The effect of CORM-2 on microglia phagocytosis}

After $6 \mathrm{~h}$ of ${ }^{\mathrm{PKH} 26+} \mathrm{RBCs}$ (small cells with red fluorescence) and microglia co-culture, the phenomenon of ${ }^{\mathrm{PKH}} 26{ }^{+} \mathrm{RBC}$ s were wrapped in microglia (large cells with red fluorescence) were found under a 20 -fold inverted fluorescence microscope. The number of ${ }^{\mathrm{PKH} 26+} \mathrm{RBC}$ (small red spots, white arrow) and the number of microglia who swallowed ${ }^{\mathrm{PKH} 26+} \mathrm{RBC}$ (large red spots, red arrow) in the co-culture model were calculated, respectively, using a blood-cell counting board at the 24 th $h$ and the $72 \mathrm{nd} h$ after CORM-2 treatment.

We noticed that the number of microglia who swallowed ${ }^{\mathrm{PKH} 26+} \mathrm{RBCs}$ (Red arrow) were gradually increasing from $24 \mathrm{~h}$ through $72 \mathrm{~h}$ after co-culture in both microglia $+{ }^{\mathrm{PKH} 26+} \mathrm{RBC}$ group and microglia + $\mathrm{PKH}^{26+} \mathrm{RBCs}+\mathrm{CORM}-2$ group. Which indicated that more and more RBCs were swallowed by microglia in all the two cell-groups over time.

More importantly, we found that the number of microglia who swallowed ${ }^{\mathrm{PKH} 26+}{ }^{\mathrm{RBC}}$ (Red arrow) increased more remarkably in CORM-2 group than that in non-CORM-2 group at both the 24th $\mathrm{h}$ and the 72nd h, meanwhile, the number of ${ }^{\mathrm{PKH} 26+}{ }^{\mathrm{RBC}}$ (White arrow) decreased more remarkably in CORM-2 group than that in non-CORM-2 group accordingly at the same time points (Fig. 2), all of which indicated that CORM-2 increased the microglia phagocytosis to RBCs. 


\section{The effect of CORM-2 on HO-1, NF-KB p65, and IL-1 $\beta$ expression}

RT-qPCR revealed that at the $1 \mathrm{st}$ day, the levels of HO-1 in all cell-groups were as high as the same, without showed statistic difference, while the levels of IL-1 $\beta$ and NF-KBp65 in microglia $+{ }^{\mathrm{PKH} 26+} \mathrm{RBCs}$, and microglia $+{ }^{\mathrm{PKH} 26+} \mathrm{RBCs}+\mathrm{CORM}-2$ groups were remarkably higher than that in microglia group, which revealed that RBCs induced inflammation. However, the levels of IL-1 $\beta$ and NF-KBp65 in microglia + $\mathrm{PKH} 26+\mathrm{RBCs}$ group were higher than that in microglia + PKH26 + RBCs + CORM-2 group, which revealed that CORM-2 can inhibit inflammation and consume HO-1. At the 3rd d, HO-1 mostly increased in microglia $+{ }^{\mathrm{PKH} 26+} \mathrm{RBCs}+\mathrm{CORM}-2$ group, and decreased in microglia $+{ }^{\mathrm{PKH} 26+} \mathrm{RBC}$ group, all of which revealed that CORM-2 increased HO-1 expression. IL-1 $\beta$ and NF-KB decreased remarkably at the 3st day and the 5th day in microglia $+{ }^{\mathrm{PKH} 26+} \mathrm{RBC}$ + CORM-2 group (Fig. 3).

Western blot analysis revealed that at the 1st day, H0-1 protein expression showed no significant differences among all cell-groups; whereas, at the 3rd day and the 5th day, the HO-1 protein expression was most remarkably the microglia + ${ }^{\mathrm{PKH} 26+} \mathrm{RBC}+\mathrm{CORM}-2$ group, which indicated that CORM-2 significantly increased HO-1 protein expression. At the same time, CORM-2 significantly reduced IL-1 $\beta$ and NF-KB protein expression (Fig. 4).

Immunofluorescence showed no significant differences of H0-1 expression on the 1st day and the 3rd day. On the 5th day, higher level of HO-1 expression was found in microglia $+{ }^{\mathrm{PKH}}{ }^{26+} \mathrm{RBC}+\mathrm{CORM}-2$ group; and the lower levels of IL-1 $\beta$ and NF-KB p65 were also in microglia + ${ }^{\mathrm{PKH} 26+} \mathrm{RBC}+\mathrm{CORM}-2$ group, when compared with other two groups (Fig. 5). Which revealed that CORM-2 could increase HO-1 and decrease $L-1 \beta$ and NF-KB p65 expression.

\section{HbCO levels in the co-culture model}

As shown in Fig. 6, there was no significant difference of $\mathrm{HbCO}$ levels among cell-groups at the 1 st day. However, compared with the microglia $+{ }^{\mathrm{PKH} 26+} \mathrm{RBC}$ group, $\mathrm{HbCO}$ in microglia $+{ }^{\mathrm{PKH} 26+} \mathrm{RBC}$ + $\mathrm{CORM}-2$ group showed a mildly decrease.

\section{Discussion}

ICH-induced brain injury can be divided into two phases: primary injury caused by the mass effect of intraparenchymal hematoma and secondary injury caused by oxidative stress and neuroinflammation in the perihematomal area[17, 18].

Hematoma and its degradation products may activate post-ICH inflammatory responses in the perihematomal region[19]. Effective hematoma removal is crucial for regulating inflammation and functional recovery[20]. Microglia are crucial for tissue repair involving hematoma and damaged-cell phagocytosis post-ICH; moreover, they exert anti-inflammatory and pro-inflammatory effects. Microglia 
are innate immune cells in the brain and are considered as the first kind of non-neuronal cells to respond to various acute brain injuries, including $\mathrm{ICH}[21,22]$. Therefore, this study established a microglia/RBC co-culture model in vitro to explore the microglial involvement in $\mathrm{ICH}$.

Hematoma removal and absorption are essential for $\mathrm{ICH}$ recovery and are clinically achieved through craniotomy or minimally invasive hematoma removal surgery and drug treatment. Moreover, the quality of clinical outcomes is positively correlated with the speed of hematoma absorption[23]. Therefore, promoting endogenous hematoma absorption has become a novel ICH treatment strategy[24]. Microglia, which are effector cells of immune and inflammatory responses in the central nervous system, can clear hematomas and protect nerve cells by phagocytizing RBCs and dissolved RBC components. In the LPSinduced inflammatory injury model, the $\mathrm{HO}-1 / \mathrm{CO}$ system was found to promote microglia migration, accelerate microglial phagocytosis, and protect central nervous system[25]. In the microglia/erythrocyte co-culture model in this study, the microglia $+{ }^{\mathrm{PKH} 26+} \mathrm{RBCs}+\mathrm{CORM}-2$ group was confirmed have more strong efficacy on increasing HO-1and decreasing IL-1 $\beta$ and NF-KB p65. All of which indicated that CORM-2 could increase microglial phagocytosis of RBCs and inhibit bleeding-induced inflammation.

In addition to accelerating hematoma absorption, it is important to regulate the inflammatory response around the hematoma. Neuronal injury and related neurological outcomes are dependent on a delicate balance between pro-inflammatory and anti-inflammatory mediators[26]. Endogenous $\mathrm{CO}$ is mainly oxidized by heme, widely involved in cardiovascular diseases, respiratory lesions, and other physiological /pathophysiological processes, has been confirmed with anti-inflammatory, anti-apoptotic, and antioxidative effects[13, 27].

CO was confirmed could inhibit the expression of inflammatory factors, such as IL-1 $\beta$, and macrophage inflammatory protein $1 \beta$, and reduce inflammatory injury $[28,29]$. The HO-1/CO system can inhibit TNF-amediated inflammatory response [27], and CORM-2 can not only up-regulate $\mathrm{HO}-1$ but also induce the cytoprotective effect of $\mathrm{HO}-1[30]$. This study revealed the mechanism underlying erythrophagocytosis modulation by the $\mathrm{HO}-1 / \mathrm{CO}$ system and neuroprotection by microglia. Activated microglia can release reactive oxygen species, which cause protein oxidation, membrane lipid peroxidation, enzyme inactivation, and DNA damage [31]. However, Mayne reported that decreased microglial TNF-a expression reduced neuronal apoptosis around the hematoma and improved the neurobehavioral score[32]. Our findings confirmed that CORM-2 effectively increased HO-1 expression, as well as inhibited NF-KB p65 and IL-1 $\beta$ expression in the microglia and RBC co-culture model, which suggested that CORM-2 could induce the microglia anti-inflammatory effect. However, it remains unclear whether CORM-2 inhibits the NF-KB signaling pathway by suppressing NF-KB p65 subunit phosphorylation or the nuclear translocation process of the NF-KB p65 subunit. HO-1 could inhibit the production of numerous downstream inflammatory factors of NF-KB by inhibiting and promoting NF-KB p65 and nuclear factor 2-related factor (Nrf2) entry, respectively, into the nucleus. Therefore, it plays a neuroprotective effect on early injury around the cerebral hemorrhage focus in rats[8-10]. In this study, CORM-2 significantly inhibited cellular and nuclear NF-kB p65 expression, which indicates that the HO-1/CO system may enhance the microglia anti-inflammatory effect by inhibiting post-ICH nuclear translocation of NF-kB p65 subunit. 
Finally, we assessed whether CORM2 increased $\mathrm{HbCO}$ and affected oxygen metabolism. The affinity of carbon monoxide to hemoglobin is approximately 200 times greater than that of oxygen; therefore, $\mathrm{CO}$ poisoning could cause hypoxic damage. CORM-2 can slowly release CO in DMSO solution, which is convenient for controlling the $\mathrm{CO}$ release rate[33]. We found that CORM-2 adding to the microglia + RBC co-culture model did not increase the $\mathrm{HbCO}$ levels; instead, it mildly decreased $\mathrm{HbCO}$. CORM-2 could enhance microglial phagocytosis to RBCs, which decreased hemoglobin level. In this study, although there was a gradual increase in $\mathrm{CO}$ expression, $\mathrm{HbCO}$ saturation remained stable $(500-600 \mathrm{ng} / \mathrm{ml})$ after CORM-2 treatment, indicated that CORM-2 did not produce serious toxic and side effects. And also, CORM-2 did not result in excessive carboxyhemoglobin levels. Study identified that low level of $\mathrm{HbCO}$ did not insignificantly affect overall mitochondrial function and biogenetics, but resulted in a significant increasing in the basal oxygen consumption rate. Assessment of mitochondrial function with inhibitors revealed no other alterations in the oxygen consumption rate [34]. Although $\mathrm{CO}$ gas has already passed safety evaluation in phase I trials in healthy humans and possesses a backbone carrier moiety, CORM-2 should be stringently characterized from a metabolic and toxicological perspective[35]. Further study is needed to elucidate the pharmacokinetics and biology of $\mathrm{CO}$ and CORMs.

\section{Conclusions}

CORM-2 can inhibit inflammatory reactions in bleeding setting in vitro by promoting microglial phagocytosis to RBCs and decrease IL-1 $\beta$ and NF-KB; the mechanism may involve HO-1/CO system.

\section{Declarations}

\section{Funding}

This study was sponsored by the National Key R\&D Program (2017YFC1308401, 2018YF100900), the National Natural Science Foundation (81660209, 81760221, 81703960, 81960221), and the Project of Beijing Municipal Top Talent of Healthy Work (2014-2-015) of China.

\section{Conflicts of interest}

The authors have no conflicts of interest to declare.

\section{Availability of data and materials}

The datasets used and/or analyzed during the current study are available from the corresponding author on reasonable request.

\section{Authors' contributions}


ZYC,XPY and RM are responsible for the design of this study, acquisition, analysis, and interpretation of data for the work. ZCY and HYZ drafted the work; JZ, XQY, MXW and ZYC revised the draft critically for important intellectual content; ZYC provided approval for publication of the content; LH and ZYC collected the detailed information; XPY and RM had cooperation in the revision of the manuscript; all authors read and approved the final manuscript.

\section{Ethics approval}

The present study was performed in accordance with the recommendations outlined in the Guide for the Care and Use of Laboratory Animals and in accordance with the National Institutes of Health, and was approved by the Committee on the Ethics of Affiliated Hospital of Jiujiang University (2018011).

\section{Consent to participate}

Not Applicable

\section{Consent for publication}

Not Applicable

\section{References}

1. Zhou Y, Wang Y, Wang J, Anne SR, Yang QW. Inflammation in intracerebral hemorrhage: from mechanisms to clinical translation. Prog Neurobiol 2014; 115:25-44.

2. Toh SQ, Glanfield A, Gobert GN, Jones MK. Heme and blood-feeding parasites: friends or foes? Parasit Vectors 2010; 3:108.

3. Askenase MH, Sansing LH. Stages of the Inflammatory Response in Pathology and Tissue Repair after Intracerebral Hemorrhage. Semin Neurol 2016; 36:288-97.

4. Righy C, Bozza MT, Oliveira MF, Bozza FA. Molecular, Cellular and Clinical Aspects of Intracerebral Hemorrhage: Are the Enemies Within? Curr Neuropharmacol 2016; 14:392-402.

5. Sun Z, Wu K, Gu L, Huang L, Zhuge Q, Yang S, et al. IGF-1R stimulation alters microglial polarization via TLR4/NF-kappaB pathway after cerebral hemorrhage in mice. Brain Res Bull 2020; 164:221-234.

6. Lan X, Han X, Li Q, Yang QW, Wang J. Modulators of microglial activation and polarization after intracerebral haemorrhage. Nat Rev Neurol 2017; 13:420-433.

7. Kaiser S, Frase S, Selzner L, Lieberum JL, Wollborn J, Niesen WD, et al. Neuroprotection after Hemorrhagic Stroke Depends on Cerebral Heme Oxygenase-1. Antioxidants (Basel) 2019; 8.

8. Yin XP, Chen ZY, Zhou J, Wu D, Bao B. Mechanisms underlying the perifocal neuroprotective effect of the Nrf2-ARE signaling pathway after intracranial hemorrhage. Drug Des Devel Ther 2015; 9:5973-86. 
9. Yin XP, Zhou J, Wu D, Chen ZY, Bao B. Effects of that ATRA inhibits Nrf2-ARE pathway on glial cells activation after intracerebral hemorrhage. Int J Clin Exp Pathol 2015; 8:10436-43.

10. Yin XP, Wu D, Zhou J, Chen ZY, Bao B, Xie L. Heme oxygenase 1 plays role of neuron-protection by regulating Nrf2-ARE signaling post intracerebral hemorrhage. Int J Clin Exp Pathol 2015; 8:10156-63.

11. Subedi L, Lee JH, Yumnam S, Ji E, Kim SY. Anti-Inflammatory Effect of Sulforaphane on LPSActivated Microglia Potentially through JNK/AP-1/NF-kappaB Inhibition and Nrf2/HO-1 Activation. Cells 2019; 8.

12. Scheiblich $\mathrm{H}$, Bicker $\mathrm{G}$. Regulation of microglial migration, phagocytosis, and neurite outgrowth by HO-1/CO signaling. Dev Neurobiol 2015; 75:854-76.

13. Joshi HP, Kim SB, Kim S, Kumar H, Jo MJ, Choi H, et al. Nanocarrier-mediated Delivery of CORM-2 Enhances Anti-allodynic and Anti-hyperalgesic Effects of CORM-2. Mol Neurobiol 2019; 56:55395554.

14. Shao L, Liu C, Wang S, Liu J, Wang L, Lv L, et al. The impact of exogenous CO releasing molecule CORM-2 on inflammation and signaling of orthotopic lung cancer. Oncol Lett 2018; 16:3223-3230.

15. Jiang L, Fei D, Gong R, Yang W, Yu W, Pan S, et al. CORM-2 inhibits TXNIP/NLRP3 inflammasome pathway in LPS-induced acute lung injury. Inflamm Res 2016; 65:905-915.

16. Garcia-Arnandis I, Guillen MI, Gomar F, Castejon MA, Alcaraz MJ. Control of cell migration and inflammatory mediators production by CORM-2 in osteoarthritic synoviocytes. PLoS One 2011; 6:e24591.

17. Schipper HM, Song W, Tavitian A, Cressatti M. The sinister face of heme oxygenase-1 in brain aging and disease. Prog Neurobiol 2019; 172:40-70.

18. Zhang CY, Ren XM, Li HB, Wei W, Wang KX, Li YM, et al. Simvastatin alleviates inflammation and oxidative stress in rats with cerebral hemorrhage through Nrf2-ARE signaling pathway. Eur Rev Med Pharmacol Sci 2019; 23:6321-6329.

19. Wilhelmus MM, Boelens WC, Kox M, Maat-Schieman ML, Veerhuis R, de Waal RM, et al. Small heat shock proteins associated with cerebral amyloid angiopathy of hereditary cerebral hemorrhage with amyloidosis (Dutch type) induce interleukin-6 secretion. Neurobiol Aging 2009; 30:229-40.

20. Shao A, Zhu Z, Li L, Zhang S, Zhang J. Emerging therapeutic targets associated with the immune system in patients with intracerebral haemorrhage (ICH): From mechanisms to translation. EBioMedicine 2019; 45:615-623.

21. Lan X, Liu R, Sun L, Zhang T, Du G. Methyl salicylate 2-0-beta-D-lactoside, a novel salicylic acid analogue, acts as an anti-inflammatory agent on microglia and astrocytes. $J$ Neuroinflammation 2011; 8:98.

22. Wang J. Preclinical and clinical research on inflammation after intracerebral hemorrhage. Prog Neurobiol 2010; 92:463-77.

23. Ziai WC, Carhuapoma JR. Intracerebral Hemorrhage. Continuum (Minneap Minn) 2018; 24:16031622. 
24. Aronowski J, Zhao X. Molecular pathophysiology of cerebral hemorrhage: secondary brain injury. Stroke 2011; 42:1781-6.

25. Ogawa T, Hanggi D, Wu Y, Michiue H, Tomizawa K, Ono S, et al. Protein therapy using hemeoxygenase-1 fused to a polyarginine transduction domain attenuates cerebral vasospasm after experimental subarachnoid hemorrhage. J Cereb Blood Flow Metab 2011; 31:2231-42.

26. Thonhoff JR, Simpson EP, Appel SH. Neuroinflammatory mechanisms in amyotrophic lateral sclerosis pathogenesis. Curr Opin Neurol 2018; 31:635-639.

27. Lin CC, Hsiao LD, Cho RL, Yang CM. CO-Releasing Molecule-2 Induces Nrf2/ARE-Dependent Heme Oxygenase-1 Expression Suppressing TNF-alpha-Induced Pulmonary Inflammation. J Clin Med 2019; 8.

28. Xue J, Habtezion A. Carbon monoxide-based therapy ameliorates acute pancreatitis via TLR4 inhibition. J Clin Invest 2014; 124:437-47.

29. Qin W, Zhang J, Lv W, Wang X, Sun B. Effect of carbon monoxide-releasing molecules II-liberated CO on suppressing inflammatory response in sepsis by interfering with nuclear factor kappa $\mathrm{B}$ activation. PLoS One 2013; 8:e75840.

30. Sun B, Sun Z, Jin Q, Chen X. CO-releasing molecules (CORM-2)-liberated CO attenuates leukocytes infiltration in the renal tissue of thermally injured mice. Int J Biol Sci 2008; 4:176-83.

31. Colton CA, Gilbert DL. Microglia, an in vivo source of reactive oxygen species in the brain. Adv Neurol 1993; 59:321-6.

32. Mayne M, Ni W, Yan HJ, Xue M, Johnston JB, Del BM, et al. Antisense oligodeoxynucleotide inhibition of tumor necrosis factor-alpha expression is neuroprotective after intracerebral hemorrhage. Stroke $2001 ; 32: 240-8$.

33. Motterlini R, Clark JE, Foresti R, Sarathchandra P, Mann BE, Green CJ. Carbon monoxide-releasing molecules: characterization of biochemical and vascular activities. Circ Res 2002; 90:E17-24.

34. Reiter CE, Alayash Al. Effects of carbon monoxide (CO) delivery by a CO donor or hemoglobin on vascular hypoxia inducible factor 1alpha and mitochondrial respiration. FEBS Open Bio 2012; 2:1138.

35. Motterlini R, Otterbein LE. The therapeutic potential of carbon monoxide. Nat Rev Drug Discov 2010; 9:728-43.

\section{Figures}




\section{DAPI}

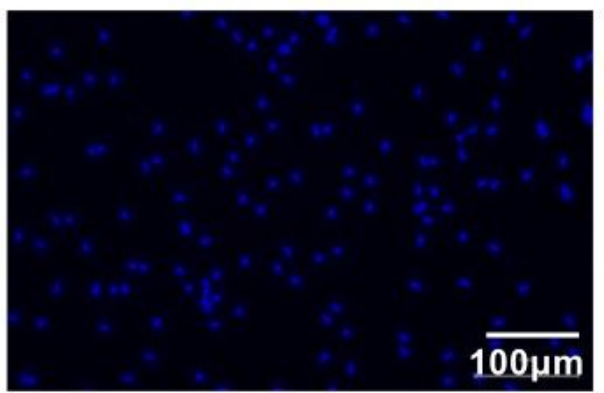

CD11b/c

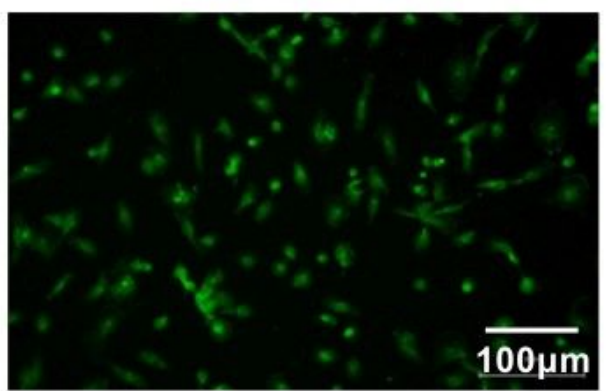

Merge

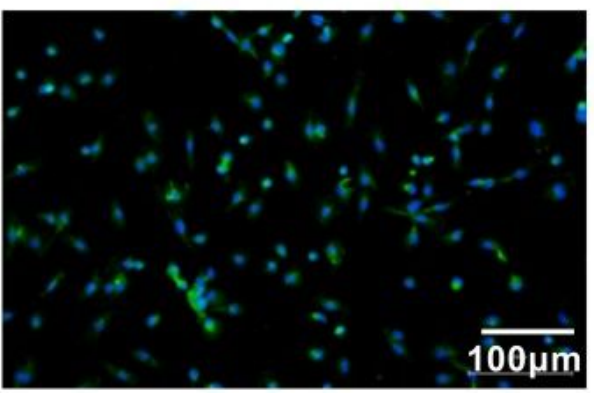

\section{Figure 1}

Identification of microglia

0 day
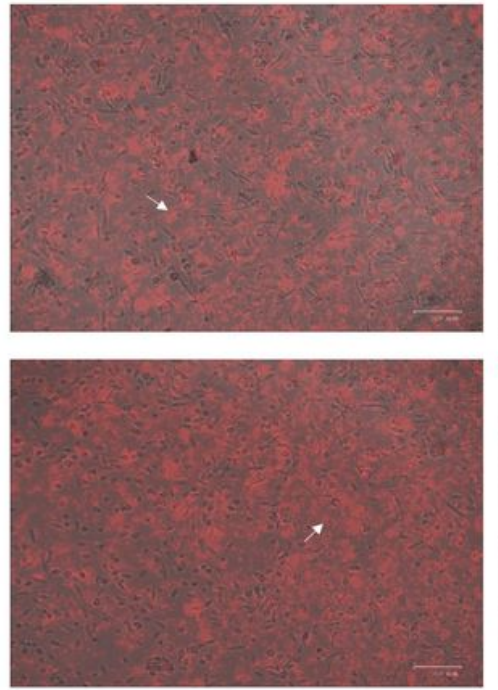

$2 \mathrm{~A}$

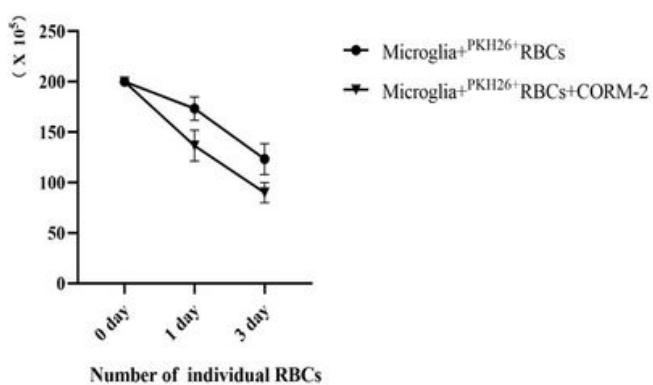

1 day
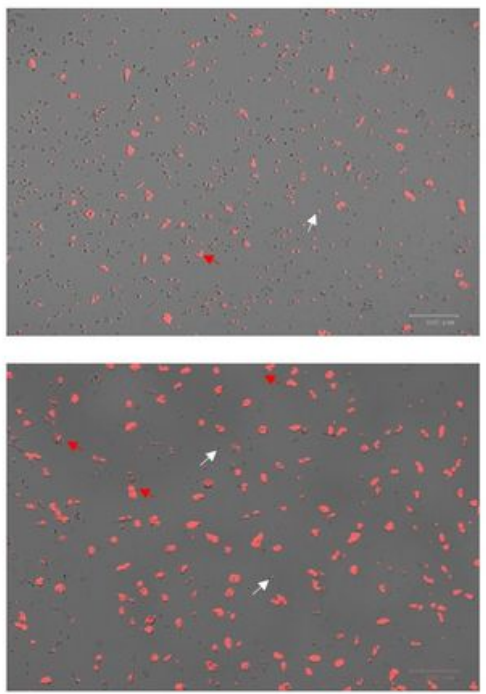

2B

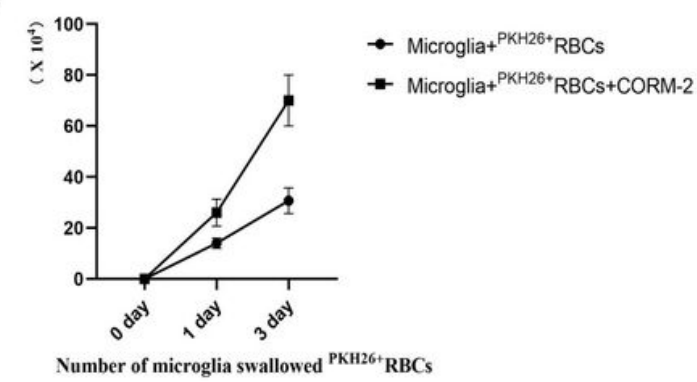

Figure 2

Microglia and PKH26+RBCs RBC density in the co-cultures White arrow indicates the PKH26+RBCs, Red arrow indicates the microglia swallowed RBCs. 


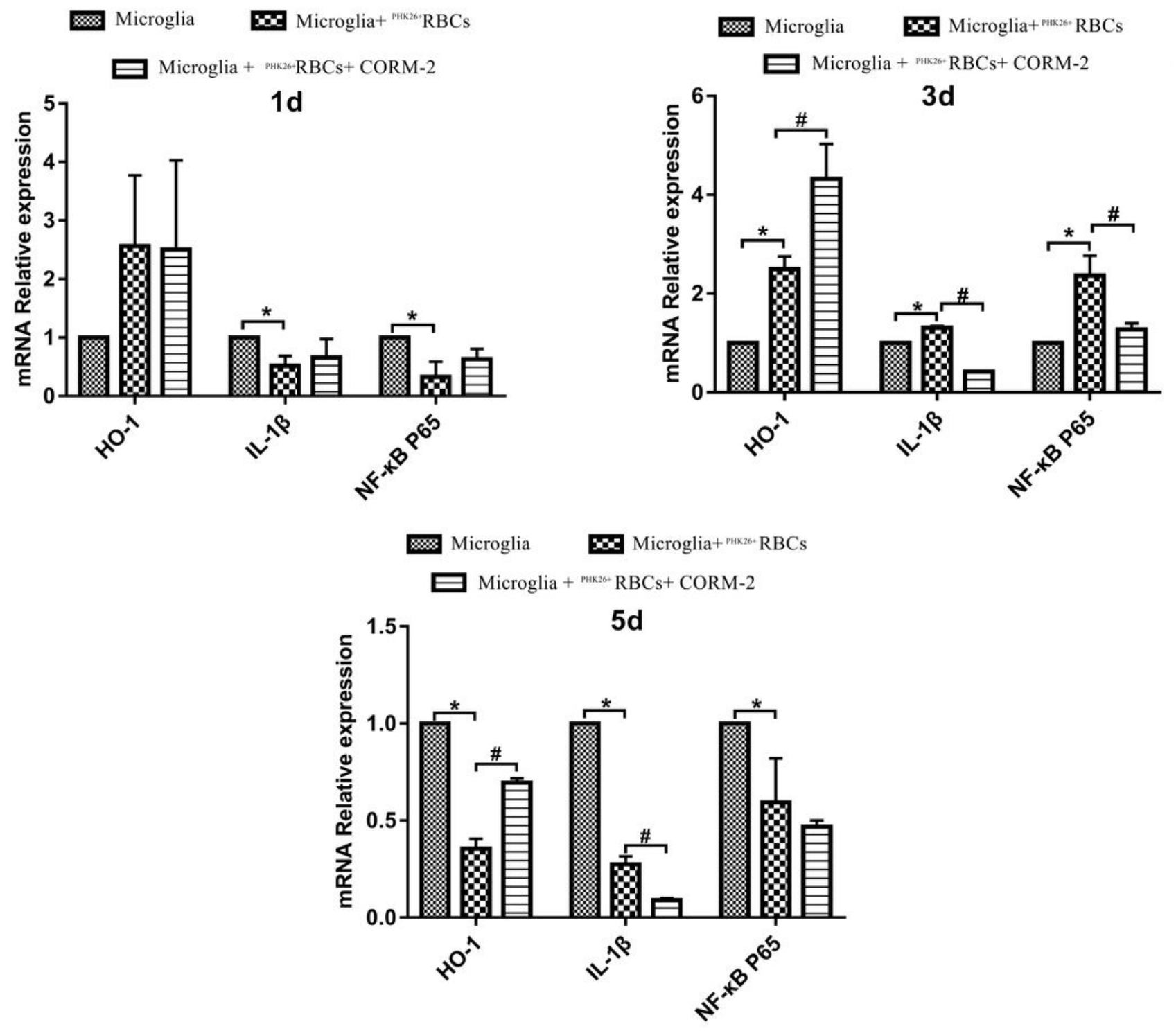

Figure 3

RT-qPCR about HO-1, NF-kB p65, and IL-1 $\beta$ mRNA expression * $\mathrm{p}<0.05$ Microglia vs. Microglia+ $\mathrm{PKH} 26+\mathrm{RBC}$ group; $\# \mathrm{p}<0.05$, Microglia $+\mathrm{PKH} 26+\mathrm{RBC}$ group vs. Microglia + PKH26+RBCs+ CORM-2 group. 

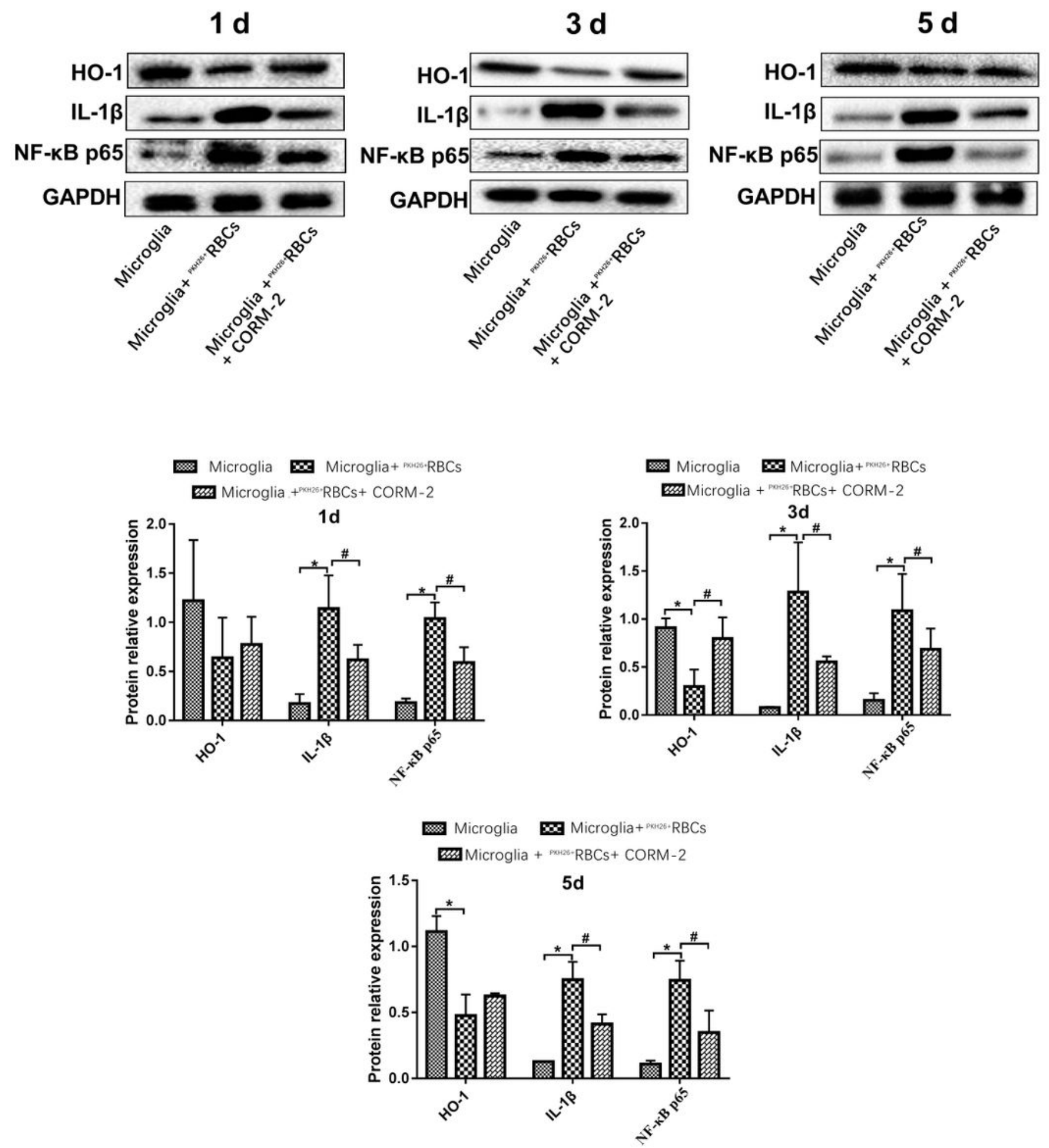

Figure 4

Western blot about HO-1, NF-kB p65, and IL-1 $\beta$ protein expression ${ }^{*} \mathrm{p}<0.05$ Microglia vs. Microglia+ $\mathrm{PKH} 26+\mathrm{RBC}$ group; $\# \mathrm{p}<0.05$, Microglia+ $\mathrm{PKH} 26+\mathrm{RBC}$ group vs. Microglia + PKH26+RBCs+ CORM-2 group. 
HO-1
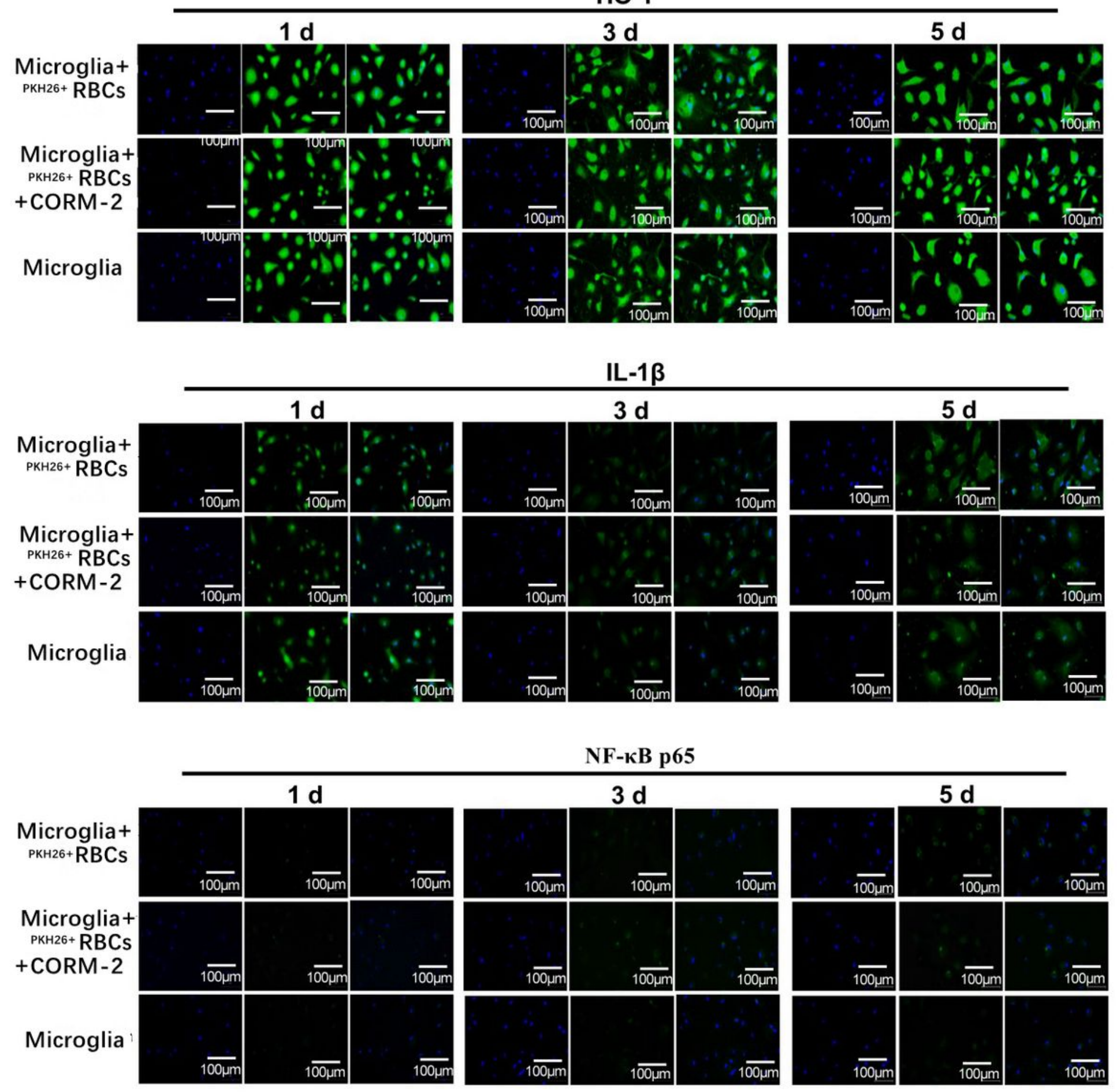

Figure 5

Immunofluorescence about HO-1, NF-KB p65, and IL-1 $\beta$ expression * $<<0.05$ Microglia vs. Microglia+ PKH26+RBCs group; \#p < 0.05, Microglia+ PKH26+RBCs group vs. Microglia + PKH26+RBCs+ CORM-2 group. 


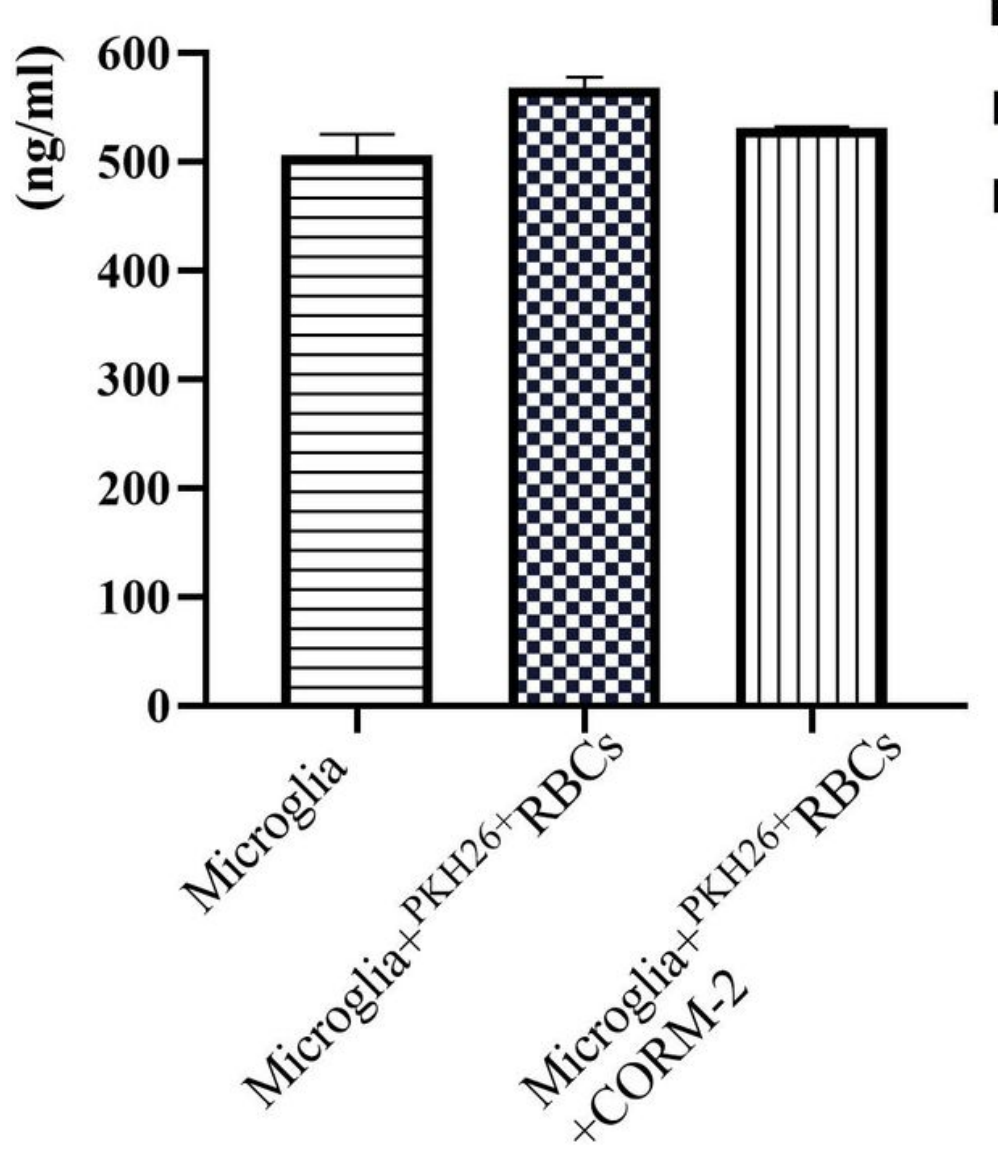

Actual HbCO content
口 Microglia

Microglia $+{ }^{\mathrm{PKH} 26+}{ }^{\mathrm{RBCs}}$

microglia $+{ }^{\mathrm{PKH} 26+}{ }^{\mathrm{RBCs}}+\mathrm{CORM}-2$

Figure 6

ELISA results about $\mathrm{HbCO}$ levels in cell supernatant 